\title{
UCRL-PROC-218022
}

LAWRENCE LIVERMORE N A TIO N A L LABORATORY

\section{Prospects for CUORE and Latest Results from CUORICINO}

E. B. Norman

January 9, 2006

\section{PANIC05}

Santa Fe, NM, United States

September 23, 2005 through September 28, 2005 
This document was prepared as an account of work sponsored by an agency of the United States Government. Neither the United States Government nor the University of California nor any of their employees, makes any warranty, express or implied, or assumes any legal liability or responsibility for the accuracy, completeness, or usefulness of any information, apparatus, product, or process disclosed, or represents that its use would not infringe privately owned rights. Reference herein to any specific commercial product, process, or service by trade name, trademark, manufacturer, or otherwise, does not necessarily constitute or imply its endorsement, recommendation, or favoring by the United States Government or the University of California. The views and opinions of authors expressed herein do not necessarily state or reflect those of the United States Government or the University of California, and shall not be used for advertising or product endorsement purposes. 


\title{
Prospects for CUORE and Latest Results from CUORICINO
}

\author{
Eric B. Norman \\ N-Division, Lawrence Livermore National Laboratory \\ Livermore, CA 94551 U. S. A. \\ On behalf of the CUORE and CUORICINO collaborations
}

\begin{abstract}
The present status of the Cryogenic Underground Observatory for Rare Events (CUORE) is presented along with the latest results from its operating prototype, CUORICINO.
\end{abstract}

Keywords: double beta decay, neutrino mass, cryogenic detectors

PACS: 14.40.Pq, 23.40.-s, 23.40.Bw, 27.60.+j

CUORE (Cryogenic Underground Observatory for Rare Events) is a proposed next generation experiment designed to search for the neutrinoless DBD of ${ }^{130} \mathrm{Te}$ using a bolometric technique. The source/detector will be composed of 988 $5 \times 5 \times 5-\mathrm{cm}$ single crystals of $\mathrm{TeO}_{2}$ all housed in a common dilution refrigerator and operated at a temperature of $8-10 \mathrm{mK}$. The total mass of ${ }^{130} \mathrm{Te}$ contained in CUORE will be approximately $203 \mathrm{~kg}$. Attached to each crystal will be one or more neutron-transmutation doped (NTD) germanium thermistors that will measure the small temperature rise produced in a crystal when radiation is absorbed. A schematic illustration of the CUORE detector is shown in Figure 1. Details about the $\mathrm{TeO}_{2}$ cryogenic detector are contained in a NIM A paper ${ }^{1}$ and the physics potential of CUORE is described in a recent article in Astroparticle Physics. ${ }^{2}$ A complete description of the CUORE project is also available online. ${ }^{3}$ The estimated sensitivity of CUORE illustrated in Figure 2 is sufficient to cover essentially all of the so-called inverted mass hierarchy region deduced from $v$ oscillation experiments. There are several compelling reasons to study ${ }^{130} \mathrm{Te}$ DBD. The $\beta \beta$ decay of ${ }^{130} \mathrm{Te}$ has been observed in geo-chemical experiments. Thus, a direct laboratory measurement of the $2 v \beta \beta$ decay rate will provide an excellent calibration for $0 v$-DBD. Second, because of its large decay energy and large expected nuclear matrix element, the half-life of ${ }^{130} \mathrm{Te}$ is predicted to be shorter than that of a number of other candidate isotopes. Third, based on the sensitivity needed to reach the mass scales inferred from the above-mentioned oscillation experiments, the ${ }^{130} \mathrm{Te}$ experiment can be done utilizing the natural abundance of ${ }^{130} \mathrm{Te}(34 \%)$, without the time and expense of obtaining separated isotopes. Of all the proposed next generation DBD experiments, only CUORE can reach the needed sensitivity without isotopic enrichment. 
One of the great strengths of CUORE is its modular nature and the fact that many isotopes can be incorporated in a bolometer. Once CUORE is constructed, one could remove a number of crystals from the center of the array and replace them with crystals containing other materials of interest. The rest of CUORE could then be used as an anti-coincidence shield, which would provide the lowest background environment in the world in which to search for rare events. Exquisitely sensitive searches could be made for rare nuclear decays or for exotic processes such as the decay of the electron. CUORE has been approved by the Scientific Committee of the Gran Sasso National Laboratory (LNGS) in Italy and has been assigned space in this facility.

Array of 988 crystals:

19 towers of 52 crystals/tower.

$\mathrm{M}=0.78$ ton of $\mathrm{TeO}_{2}$

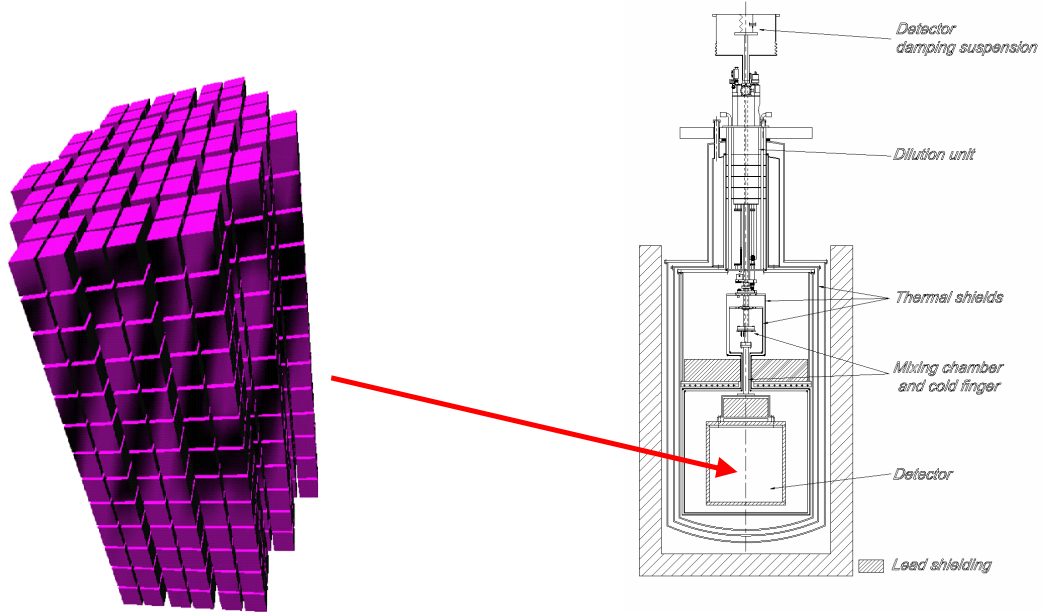

FIGURE 1. Schematic drawing of the CUORE detector

\section{CUORE Sensitivity}

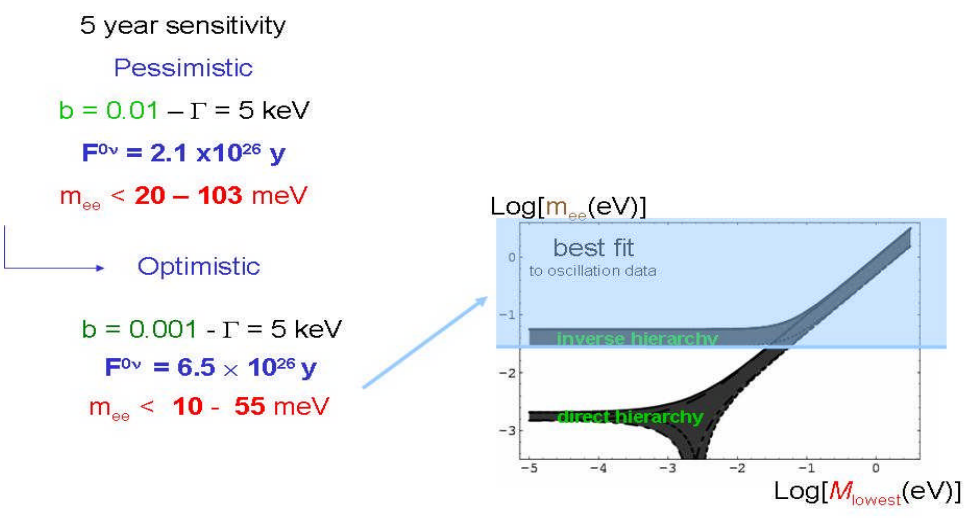

FIGURE 2. Estimated sensitivity of CUORE from 5 years of running. 
A prototype experiment, CUORICINO, which consists of $62 \mathrm{TeO}_{2}$ crystals, is now running at the LNGS. CUORICINO is presently the largest operating double beta decay experiment in the world and has recently published its first physics results $^{4,5}$ - a limit on neutrino mass only slightly less stringent than those obtained from previous ${ }^{76} \mathrm{Ge}$ experiments.

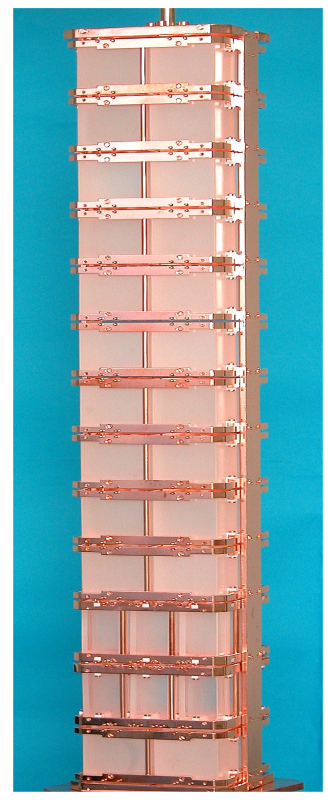

FIGURE 3. CUORICINO array currently operating at the LNGS.

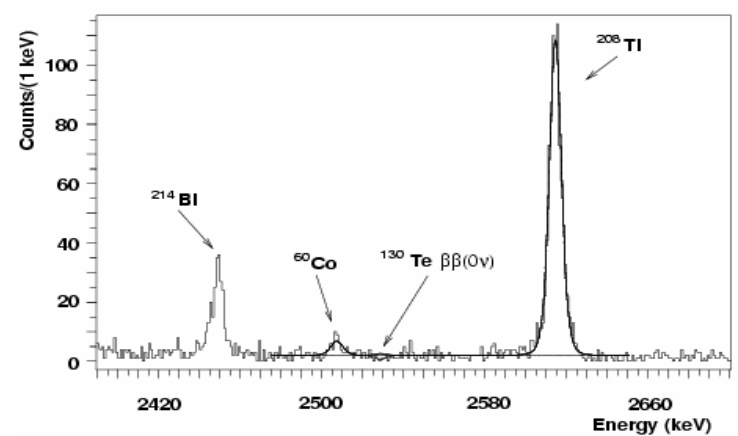

FIGURE 4. Background spectrum obtained from CUORICINO in an exposure of 10.85 kg-y. The energy resolution measured at 2615 $\mathrm{keV}=9.2 \pm 0.5 \mathrm{keV}(\mathrm{FWHM})$. The background in the $0 v \beta \beta$ region $=0.18 \pm 0.01$ counts $/(\mathrm{keV} / \mathrm{kg} / \mathrm{y})$. No peak is observed at the expected position of $0 v \beta \beta$ and a limit of $\mathrm{t}_{0 \mathrm{v} 1 / 2}$ $>1.8 \times 10^{24}$ y at $90 \%$ C.L. has been obtained. The inferred limit on neutrino mass derived from this results is: $\mathrm{m}_{v}<0.2-1.1 \mathrm{eV}$, depending on the assumed nuclear matrix elements

Since 1990, members of the CUORE collaboration have developed and operated cryogenic $\mathrm{TeO}_{2}$ bolometers ranging in mass from 73 grams up to the present CUORICINO mass of $62 \mathrm{~kg}$. Thus, CUORE is a very natural and reachable extension based upon what we have already demonstrated.

\section{ACKNOWLEDGMEN TS}

This work was performed under the auspices of the U. S. Department of Energy by the University of California, Lawrence Livermore National Laboratory under Contract number W-7405-Eng-48.

\section{REFERENCES}

1. C. Arnaboldi et al., Nucl. Instrum. \& Meth. A 518, 775 (2004).

2. C. Arnaboldi et al., Astroparticle Physics 20, 91(2003).

3. R. Ardito et al., http://xxx.lanl.gov/hep-ex/0501010.

4. C. Arnaboldi et al., Phys. Lett. B 584, 260(2004).

5. C. Arnaboldi et al. Phys. Rev. Lett. 95, 142501 (2005) 